\title{
25 Research Square \\ Multi-channel NIR/SWIR Lanthanide Nanoprobe for Forensic Gender Identification of Fingerprints
}

\author{
Yuxin Liu \\ Max-Planck Institute for Colloids and Interfaces \\ Zheng Wei \\ Capital Normal University \\ Xingjun Zhu \\ Fudan University \\ Luoyuan Li \\ Capital Normal University \\ Liyi Ma \\ Capital Normal University \\ Jing Zhou ( $\nabla$ jingzhou@cnu.edu.cn ) \\ Capital Normal University https://orcid.org/0000-0002-5348-1966
}

Article

Keywords: forensic gender identification, fingerprint imaging, luminescence

Posted Date: April 30th, 2021

DOl: https://doi.org/10.21203/rs.3.rs-439647/v1

License: (c) (i) This work is licensed under a Creative Commons Attribution 4.0 International License.

Read Full License 


\section{Abstract}

Fingerprints play vital roles in modern forensic investigations by providing essential personal information. To maximize the efficiency of forensic investigations, highly efficacious protocols are urgently needed for fingerprint capture and information extraction. Herein, a novel L-amino acids (L-AA)responsive nanoprobe was rationally designed and constructed with orthogonal near-infrared (NIR) and short wave infrared (SWIR) emissions. Due to the difference in L-AA content in fingerprints between males and females, effective gender identification is also practicable by analysing the NIR/SWIR luminescence ratio. More importantly, by utilizing adhesive L-AA on latent fingerprints as a target, simultaneous washing-free NIR/SWIR ratiometric luminescence imaging and forensic gender identification of latent fingerprints are achieved with not only high spatial resolution but advanced precision. Our findings provide a new strategy for the development of a new generation of nanoprobes for simple washing-free fingerprint imaging and valid forensic gender identification.

\section{Main Text}

In modern forensic investigations, fingerprints are considered one of the most important pieces of evidence for providing specific clues to solve cases due to their unique physical structures. ${ }^{1}$ of all the types of fingerprint impressions left at crime scenes, latent fingerprints are the hardest but the most valuable to capture. ${ }^{2}$ To date, various protocols have been developed for capturing the physical structure of latent fingerprints such as dye-staining colorimetry and luminometry. ${ }^{3}$ With advanced spatial resolution and a simple procedure, luminometry is gaining popularity in latent fingerprint capture. ${ }^{4}$ However, current luminometric methods overly emphasized the importance of complete extraction of physical structure and overlooked the simultaneous extraction of chemical information, which is equally essential and complementary to physical characterization in analysis. ${ }^{5-8}$ The rough development of simultaneous extraction should be ascribed to the lack of both knowledge to chemical characterization and appropriate probes.

Recent researches concerning chemical components of fingerprints brought great opportunity to this field. ${ }^{9,} 10$ Apart from fatty acids and inorganic salts, L-amino acids (L-AA) are the other major components in fingerprints. ${ }^{11}$ Notably, it has been demonstrated that the content of L-AA in female fingerprints is higher than in male fingerprints (Supplementary Table 1), which inspires us to consider the possibility of simultaneous latent fingerprint capture and gender identification by an L-AA-responsive luminometry protocol. ${ }^{12-14}$ Aiming to this purpose, a rationally designed probe is required, which should not only correctly indicate the amount of L-AA but minimize the signal interferes from non-specific absorption on background substrate.

In view of this, based on our expertise in construction of multi-channel lanthanide nanoprobe, an L-AAresponsive nanoprobe and corresponding ratiometric luminometry was fabricated based on the mechanisms of Förster resonance energy transfer (FRET) and the inner filter effect (IFE). Lanthanide- 
based nanophosphor, with dual orthogonal $800 \mathrm{~nm}$ near-infrared (NIR) and $1460 \mathrm{~nm}$ short wave infrared (SWIR) emission channels, was chosen as the luminescence donor (Figure 1a). For L-AA response, L-AA oxidase (L-AAO) was used to convert L-AA to hydrogen peroxide $\left(\mathrm{H}_{2} \mathrm{O}_{2}\right)$ via a catalytic oxidation reaction. The produced $\mathrm{H}_{2} \mathrm{O}_{2}$ then oxidized reduced silicomolybdate ( $\mathrm{rSiMo}$ ), a novel redox luminescence acceptor, to its non-reductive state (SiMo). In this process, the FRET and IFE between lanthanide-based nanophosphor and rSiMo will be reduced, resulting in recovery of NIR and the invariant of SWIR. The orthogonal emission channels ensure the reliability of both imaging and biodetection, which is confirmed in our previous works as an advantage of multi-channel lanthanide nanoprobes. ${ }^{15-17}$ Therefore, this method is applicable for the efficacious capture and identify of fingerprints by analysing the NIR/SWIR luminescence ratio or washing-free NIR/SWIR ratiometric luminescence imaging.

To confirm the applicability of this design, the core-shell $\mathrm{NaLuF}_{4}: \mathrm{Yb}, \mathrm{Tm}_{\mathrm{NNaLuF}}$ nanophosphor was prepared and then functionalized with SiMo (Tm-SiMo) using a layer-by-layer coating procedure, respectively (Supplementary Figure 1). Both powder X-ray diffraction patterns and the ${ }^{95}$ Mo nuclear magnetic resonance spectrum illustrated the existence of amorphous a-Keggin SiMo in the nanoprobe (Supplementary Figure 2a,b). Next, Tm-SiMo was reduced by a chemical reducing process to form rSiMo. Transmission electron microscopy images suggested that Tm-rSiMo had a near-spherical shape with an average diameter of $\sim 67.2 \mathrm{~nm}$ (Figure 1b), while energy dispersion X-ray surface mapping further demonstrated the core-shell structure of the designed nanoprobe (Figure 1c). Different to Tm-SiMo, TmrSiMo possessed strong absorbance in the NIR spectral region, which contributed to the localized surface plasmon resonance caused by intervalence charge transfer from $\mathrm{Mo}^{\mathrm{V}}$ to $\mathrm{Mo}^{\mathrm{VI}}$ (Supplementary Figure $2 \mathrm{c}, \mathrm{d})$. Due to the FRET and IFE between the lanthanide-based nanophosphor and rSiMo, NIR luminescence (centred at $800 \mathrm{~nm},{ }^{3} \mathrm{H}_{4} \rightarrow{ }^{3} \mathrm{H}_{6}$ ) of Tm-rSiMo was selectively quenched. The L-AAO was then modified on Tm-rSiMo to obtain the final L-AA-responsive nanoprobe (Tm-rSiMo-AAO). It was observed that the hydrodiameter of Tm-rSiMo-AAO was larger than pre-modification, demonstrating the absorption of L-AAO on Tm-rSiMo (Supplementary Figure 3a). It is noteworthy that the final nanoprobe was stable after long-term storage, which further ensures its application (Supplementary Figure 3b-d). The above results illustrate the successful construction of the designed nanoprobe.

Due to the L-AA catalytic nature of L-AAO, this modification process endowed the nanoprobe with L-AA catalytic capacity. The L-AA-responsive capacity of the Tm-rSiMo-AAO was then studied. Similar to other types of L-AAO, the one modified on Tm-rSiMo also had the highest catalytic activity in a weak acidic environment with a peak at $\mathrm{pH}=5.0$ (Supplementary Figure 4a). Therefore, the study of L-AA response was undertaken in this optimized weak acidic condition. In the presence of L-AA, the NIR absorbance of Tm-rSiMo-AAO decreased rapidly and significantly, while that of Tm-rSiMo barely changed, which highlights the importance of L-AAO in the L-AA response (Figure 1d,f). In contrast, the Tm-rSiMo with $\mathrm{H}_{2} \mathrm{O}_{2}$ addition possessed a similar NIR absorbance change to Tm-rSiMo-AAO with L-AA addition (Figure 1d). To further verify the mechanism of the L-AA response, cyclic voltammetry curves and the X-ray photoelectron spectra of Tm-rSiMo-AAO were determined in the presence and absence of L-AA, which indicated the reducing $\mathrm{Mo}^{\mathrm{V}}$ and increasing $\mathrm{Mo}^{\mathrm{VI}}$ amount in the presence of $\mathrm{L}-\mathrm{AA}$ and hence proved that 
rSiMo in the nanoprobe was oxidized (Supplementary Figure 4b,c). On the basis of these findings, it is reasonable to propose that the L-AA response of Tm-rSiMo-AAO consisted of two steps: 1) L-AA was oxidized by dissolved oxygen under the catalysis of L-AAO, producing a-keto acid and $\left.\mathrm{H}_{2} \mathrm{O}_{2} ; 2\right) \mathrm{H}_{2} \mathrm{O}_{2}$ oxidized rSiMo with high NIR absorbance to SiMo with low NIR absorbance. The decreased NIR absorbance of Tm-rSiMo-AAO contributed to the reduction of both FRET and IFE efficiency, resulting in the NIR luminescence recovery in the presence of L-AA (Figure 1e). However, the luminescence intensity change in the SWIR spectral region (centred at $1475 \mathrm{~nm},{ }^{3} \mathrm{H}_{4} \rightarrow{ }^{3} \mathrm{~F}_{4}$ ) was low, which was a result of the low absorbance of either rSiMo or SiMo in the corresponding region (Supplementary Figure 4d). Therefore, due to its outstanding selectivity for L-AA (Supplementary Figure 5), Tm-rSiMo-AAO is a promising nanoprobe for L-AA-responsive luminometry and quantification.

The L-AA in actual fingerprint samples was then tested using Tm-rSiMo-AAO. For gender identification, two parallel procedures were proposed: 1) extraction of L-AA from fingerprints, followed by the addition of Tm-rSiMo-AAO, measurement of luminescence spectra, and the referring threshold; 2 ) staining L-AA in fingerprints with Tm-rSiMo-AAO, followed by ratiometric luminescence imaging and signal analysis (Figure 2a). We carried out the first procedure by collecting 10 male and 10 female fingerprints and extracting L-AA using typical protocols. It was found that the NIR luminescence intensity of Tm-rSiMoAAO for female fingerprints extraction was significantly higher than that for male fingerprints extraction (Figure 2b), while the NIR/SWIR ratio of female fingerprints was also elevated compared to that of male fingerprints (Figure 2c). When utilizing the NIR/SWIR ratio of the optimal threshold, effective gender identification was achieved with a high precision rate of $97.8 \%$ (Figure $2 \mathrm{~d}$ ). These findings suggest that the gender of the fingerprint owner was accurately predicted by extracting L-AA from fingerprints and using L-AA-responsive luminometry based on Tm-rSiMo-AAO.

To simultaneously capture fingerprints and identify gender, the fingerprints were stained with Tm-rSiMoAAO following the second procedure. The NIR/SWIR ratiometric images of female and male fingerprints were obtained by the division of NIR and SWIR signals (Figure 3a). From local magnified images, the clear edge of fingerprints and physical structure with full distinct details of pattern areas are seen (Figure $3 b)$. The signal distribution indicated not only the potential of well-defined imaging with an ultrathin edge $(\sim 0.3 \mathrm{~mm})$, but also gender identification by analysing the NIR/SWIR ratio (threshold $=0.304)$, which illustrated that Tm-rSiMo-AAO is feasible for simultaneous fingerprint capture and gender identification (Figure $3 c, d$ ). It should also be mentioned that the signal-to-noise ratio of the NIR/SWIR ratiometric image was obviously higher than either the single NIR or SWIR image (Figure 3e), which was the result of eliminating interference due to uneven staining and background luminescence in washing-free cases (Supplementary Figure 6). Furthermore, compared with the NIR and SWIR images, the NIR/SWIR ratiometric image also possessed a higher female-to-male ratio, which indicated that the NIR/SWIR ratiometric image had higher accuracy in gender identification (Figure 3f). Although the NIR mainly contributed to the L-AA response, the amount of Tm-rSiMo-AAO may also have influenced the intensity. By utilizing the SWIR as a reference, the L-AA can be quantified much more precisely, resulting in more accurate gender identification by the NIR/SWIR image. 
In conclusion, we constructed a multi-channel lanthanide nanoprobe, Tm-rSiMo-AAO, for washing-free fingerprint capture and gender identification using L-AA-responsive luminometry. The L-AAO catalyses LAA in fingerprints, produces $\mathrm{H}_{2} \mathrm{O}_{2}$, oxidizes rSiMo, and results in a reduction of NIR absorbance. Correspondingly, the NIR of Tm-rSiMo-AAO recovered and SWIR barely changed in the presence of L-AA. By specifically reacting with L-AA, the Tm-rSiMo-AAO can identify differences of L-AA content in fingerprints between males and females. Notably, using this nanoprobe, precise washing-free luminescence imaging and gender identification are simultaneously achieved on actual fingerprint samples with a well-defined edge $(\sim 0.3 \mathrm{~mm})$ and high precision rate $(>97.8 \%)$, respectively. As a result, focusing on other biomolecular characteristics of fingerprints, highly efficacious extraction and precise identification of other individual information could be enabled by rational designed luminescent nanoprobes, which will attract interest from forensic science personnel and the authentication industry. Our research not only establishes a novel procedure for both washing-free fingerprints imaging and gender identification, but also allows the development of next-generation protocols for simultaneous extraction of multiple data from a single piece of evidence.

\section{Methods}

Materials. Lanthanide oxide $\mathrm{Lu}_{2} \mathrm{O}_{3}(99.999 \%), \mathrm{Yb}_{2} \mathrm{O}_{3}(99.999 \%)$ and $\mathrm{Tm}_{2} \mathrm{O}_{3}(99.99 \%)$ are purchased from STREM Chemicals, Inc. USA. Lanthanide chlorides ( $\mathrm{LnCl}_{3}$, $\mathrm{Ln}$ : $\mathrm{Lu}, \mathrm{Yb}$ and $\left.\mathrm{Tm}\right)$ are prepared by dissolving the corresponding metal oxide in $\mathrm{HCl}$ solution at elevated temperature and then evaporating the water completely under reduced pressure. 1-octadecene (ODE), $\mathrm{NH}_{4} \mathrm{NO}_{3}$, and $\mathrm{NH}_{4} \mathrm{~F}$ are purchased from Alfa Aesar Chemical Co. Ltd. $\mathrm{Na}_{2} \mathrm{SO}_{4}, \mathrm{KCl}, \mathrm{CaCl}_{2}, \mathrm{Mg}\left(\mathrm{NO}_{3}\right)_{2}, \mathrm{NaCl}, \mathrm{ZnCl}_{2}, \mathrm{KBr}, \mathrm{Na}_{3} \mathrm{PO}_{4}, \mathrm{Na}_{2} \mathrm{CO}_{3}, \mathrm{NaOH}, \mathrm{CHCl}_{3}$, $\mathrm{H}_{2} \mathrm{SO}_{4}$ solution, $\mathrm{H}_{3} \mathrm{PO}_{4}$ solution, $\mathrm{H}_{2} \mathrm{O}_{2}$ solution, citric acid, sodium citrate, ethanol, ethyl acetate, cyclohexane, oleic acid (OA), hexadecyl trimethyl ammonium bromide (CTAB), L-amino acid oxidase (LAAO, from crotalus adamanteus, type IV), horseradish peroxidase (HRP, type VI), O-dianisidine, coomassie brilliant blue (CBB), human serum albumin (HSA), carbamide, octanoic acid (OOA), decanoic acid (DOA), palmitic acid (PTA), octadecanoic acid (ODOA), arachidic acid (ADA), a-keggin silicomolybdate (SiMo), ascorbic acid (ACA), 2-hydroxypropanoic acid (2-HA), glucose (Glc), tetraethyl orthosilicate (TEOS), $\left(\mathrm{NH}_{4}\right)_{2} \mathrm{MoO}_{4}$ and $\mathrm{L}$-amino acid (L-AA) are purchased from Sigma Aldrich. All chemical reagents are of analytical grade and are used directly without further purification. Deionized (DI) water is used throughout.

Synthesis of $\mathrm{NaLuF}_{4}: \mathrm{Yb}, \mathrm{Tm}$. In a typical experiment, a mixture of $1 \mathrm{mmol} \mathrm{LnCl}_{3}(\mathrm{Ln}: 80 \% \mathrm{Lu}, 19 \% \mathrm{Yb}$, $1 \% \mathrm{Tm}), 15 \mathrm{~mL} \mathrm{OA}$, and $15 \mathrm{~mL} \mathrm{ODE}$ are added into a $100 \mathrm{~mL}$ three-necked flask. Under the vacuum, the mixture is heated to $160{ }^{\circ} \mathrm{C}$ to form a clear solution, and then cooled to room temperature. After the solution cooling down, $0.025 \mathrm{mmol} \mathrm{NaOH}(0.1 \mathrm{~g})$ and $0.04 \mathrm{mmol} \mathrm{NH}_{4} \mathrm{~F}(0.1481 \mathrm{~g})$ are added into the flask directly and stirred for 30 minutes. The solution is slowly heated with gently stirred, degassed at 100 ${ }^{\circ} \mathrm{C}$, and then heated to $300{ }^{\circ} \mathrm{C}$ and maintained for 1 hour under the Argon atmosphere. After the solution is cooled naturally, the $\mathrm{NaLuF}_{4}: \mathrm{Yb}, \mathrm{Tm}$ are separated via centrifugation $(10000 \mathrm{rpm})$ and washed with 
ethanol/cyclohexane $(1: 1 \mathrm{v} / \mathrm{v})$ three times. The product is stored under room temperature in cyclohexane. ${ }^{18}$

Synthesis of $\mathrm{NaLuF}_{4}: \mathrm{Yb}, \mathrm{Tm}_{\mathrm{NNaLuF}}$. In a typical experiment, a mixture of $1 \mathrm{mmol} \mathrm{LuCl}, 15 \mathrm{~mL} \mathrm{OA}$, and $15 \mathrm{~mL}$ ODE are added into a $100 \mathrm{~mL}$ three-necked flask. Under the vacuum, the mixture is heated to 160 ${ }^{\circ} \mathrm{C}$ to form a clear solution, and then cooled to room temperature. After the solution cooling down, 0.025 $\mathrm{mmol} \mathrm{NaOH}(0.1 \mathrm{~g}), 0.04 \mathrm{mmol} \mathrm{NH}_{4} \mathrm{~F}(0.1481 \mathrm{~g})$, and as-prepared $\mathrm{NaLuF}_{4}: Y b, T m$ nanoparticles are added into the flask directly and stirred for 30 minutes. The solution is slowly heated with gently stirred, degassed at $100^{\circ} \mathrm{C}$, and then heated to $300^{\circ} \mathrm{C}$ and maintained for 1.5 hour under the Argon atmosphere. After the solution is cooled naturally, the $\mathrm{NaLuF}_{4}: \mathrm{Yb}, \mathrm{Tm}_{\mathrm{N}} @ \mathrm{NaLuF}_{4}$ are separated via centrifugation (10000 $\mathrm{rpm})$ and washed with ethanol/cyclohexane $(1: 1 \mathrm{v} / \mathrm{v})$ three times. The product is stored under room temperature in cyclohexane.

Synthesis of $\mathrm{NaLuF}_{4}: \mathrm{Yb}_{1} \mathrm{Tm} @ N a L u F_{4} @ \mathrm{mSiO}_{2}$. In a typical experiment, $250 \mu \mathrm{L} \mathrm{CHCl}_{3}$ containing $5 \mathrm{mg}$ as-

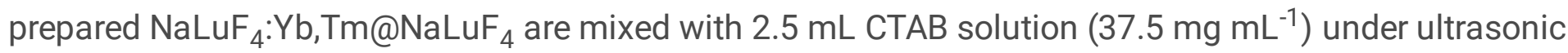
for 5 minutes. The mixture is transformed to a $100 \mathrm{~mL}$ three-necked flask and heated in $60^{\circ} \mathrm{C}$ water-bath to form a clear solution. Then, $22.5 \mathrm{~mL} \mathrm{DI}$ water is added and the solution is heated to $70^{\circ} \mathrm{C}$ before 0.3 $\mathrm{mmol} \mathrm{NaOH}(0.012 \mathrm{~g})$ is added directly. After 20 minutes stirring under the speed of $600 \mathrm{rpm}, 120 \mu \mathrm{L}$ TEOS is dropped slowly and $0.5 \mathrm{~mL}$ ethyl acetate is added within 30 seconds. Finally, the solution is continuously stirred in $70{ }^{\circ} \mathrm{C}$ water-bath for another 3 hours to enhance the stability of $\mathrm{mSiO}_{2}$ shell. After the solution is cooled naturally, the $\mathrm{NaLuF}_{4}: \mathrm{Yb}, \mathrm{Tm} @ \mathrm{NaLuF}_{4} @ \mathrm{mSiO}_{2}$ are separated via centrifugation (8000 rpm) and washed with ethanol and DI water three times, respectively. The as-obtained $\mathrm{NaLuF}_{4}: \mathrm{Yb}, \mathrm{Tm}_{\mathrm{N}} @ \mathrm{NaLuF}_{4} @ \mathrm{mSiO}_{2}$ is then suspended in $50 \mathrm{~mL}$ ethanol containing $0.3 \mathrm{~g} \mathrm{NH}_{4} \mathrm{NO}_{3}$ upon stirring for 1 hour at $60{ }^{\circ} \mathrm{C}$. The CTAB-removed $\mathrm{NaLuF}_{4}: \mathrm{Yb}, \mathrm{Tm}_{\mathrm{N}} @ \mathrm{NaLuF}_{4} @ \mathrm{mSiO}_{2}$ are separated via centrifugation $(8000 \mathrm{rpm})$ and washed with $\mathrm{DI}$ water three times. The product is stored under $4{ }^{\circ} \mathrm{C}$ in $\mathrm{DI}$ water. $^{19}$

Synthesis of $\mathrm{NaLuF}_{4}: \mathrm{Yb}, \mathrm{Tm} @ \mathrm{NaLuF}_{4} @ \mathrm{mSiO}_{2}$-SiMo (Tm-SiMo). In a typical experiment, $1 \mathrm{~mL} \mathrm{H}_{2} \mathrm{SO}_{4}$ solution is added to $5 \mathrm{~mL}$ DI water containing $5 \mathrm{mg}$ as-prepared $\mathrm{NaLuF}_{4}: \mathrm{Yb}, \mathrm{Tm}_{\mathrm{N}} @ \mathrm{NaLuF}_{4} @ \mathrm{mSiO}_{2}$. After stirring under room temperature for 10 minutes, $0.5 \mathrm{~mL}$ DI water containing $0.05 \mathrm{mmol}\left(\mathrm{NH}_{4}\right)_{2} \mathrm{MoO}_{4}$ $(0.0098 \mathrm{~g})$ is added and the mixture is then dried for $240 \mathrm{~min}$ at $100^{\circ} \mathrm{C}$ to stabilize the SiMo modification. The Tm-SiMo are separated via centrifugation $(8000 \mathrm{rpm})$ and washed with DI water three times. The product is stored under $4{ }^{\circ} \mathrm{C}$ in DI water. ${ }^{20}$

Synthesis of reduced Tm-SiMo (Tm-rSiMo). In a typical experiment, $5 \mathrm{mg}$ as-obtained Tm-SiMo are suspended in $5 \mathrm{~mL}$ DI water containing $5 \mathrm{mmol}$ ACA $(0.088 \mathrm{~g})$ upon stirring for 20 minutes at room temperature. The Tm-rSiMo are separated via centrifugation $(8000 \mathrm{rpm})$ and washed with DI water three times. The final product is stored under $4{ }^{\circ} \mathrm{C}$ in DI water. 
Modification of L-AAO on Tm-rSiMo (Tm-rSiMo-AAO). In a typical experiment, $100 \mu \mathrm{L}$ L-AAO solution (1 unit $\mathrm{mL}^{-1}$ ) is added to $5 \mathrm{~mL}$ DI water containing $5 \mathrm{mg}$ as-prepared Tm-rSiMo. After stirring under $4{ }^{\circ} \mathrm{C}$ for 120 minutes, the Tm-SiMo-AAO are separated via centrifugation $(8000 \mathrm{rpm})$ and washed with DI water three times. The product is stored under $-20^{\circ} \mathrm{C}$ in DI water.

Characterization. The sizes and morphologies of nanoprobes within layer-by-layer coating procedure are determined using a FEI Tecnai $\mathrm{G}^{2} \mathrm{~F} 30$ transmission electron microscope (TEM). Samples of the above nanoparticles are dropped on the surface of a copper grid. Energy-dispersive X-ray (EDX) mapping and selected area electron diffraction (SAED) pattern of the samples are also performed during TEM measurements. The size distribution is counted and calculated from TEM images $(a=0.90,>500$ particles are measured). Solid UV-vis-NIR absorbance spectra are obtained on a Shimadzu SolidSpec3700 UV-vis-NIR spectrophotometer. Aqueous UV-vis-NIR absorbance spectra are determined on a Shimadzu UV3600 UV-vis-NIR spectrophotometer. ${ }^{95}$ Mo nuclear magnetic resonance (NMR) spectra are determined by a Bruker Avance 600 liquid nuclear magnetic resonance spectrometer. Powder X-ray diffraction (XRD) pattern is measured with a Brucker D8 advance X-ray diffractometer from $5^{\circ}$ to $70^{\circ}(\mathrm{Cu}$ Ka radiation, $\lambda=1.54 \AA$ ). $X$-ray photoelectron spectroscopy (XPS) spectra are performed on Thermo escalab 250Xi. The sample for XRD and XPS determination is previously dried in nitrogen atmosphere at $100^{\circ} \mathrm{C}$. Electron paramagnetic resonance (EPR) spectra are determined on a Bruker A300 electron paramagnetic resonance spectrometer. Dynamic light scattering (DLS) experiments are carried out on an ALV-5000 spectrometer goniometry equipped with an ALV/LSE-5004 light scattering electronic and multiple tau digital correlator and a JDS Uniphase He-Ne laser $(533 \mathrm{~nm})$ with an output power of $22 \mathrm{~mW}$. The hydrodiameter distribution is measured at $25^{\circ} \mathrm{C}$ with a detection angle of $90^{\circ}$. Electrochemical measurements are carried out by a Bio-Logic VMP3 multichannel potentiostat at ambient temperature. Cyclic voltammetry (CV) curves are obtained by electrochemical station (Chenhua Instruments Co. $\mathrm{CHI600)}$. A three-electrode system is made up of a glassy carbon electrode (GCE, $3 \mathrm{~mm}$ in diameter) as the working electrode, an $\mathrm{Ag} / \mathrm{AgCl}$ electrode (saturated $\mathrm{KCl}$ ) as reference electrode, and a Pt wire as counter electrode. The near-infrared (NIR) and short-wave infrared (SWIR) luminescence spectra are taken on a FLS980 lifetime and steady state spectrometer (Edinburgh Instruments) equipped with an external 0$7 \mathrm{~W} 808 \mathrm{~nm}$ and $980 \mathrm{~nm}$ adjustable laser as the excitation source. Inductively coupled mass spectroscopy (ICP-MS) analysis is performed on Agilent 7500ce ICP-MS. Enzymatic activity is performed by using standard protocol and monochromator-based multifunction microplate reader (Tecan Infinite M200). NIR and SWIR images are collected by a self-developed small animal luminescence imaging system equipped with Andor iXon Ultra EMCCD (Si-based CCD) and Princeton Instrument NIRvana 640 CCD (InGaAs-based CCD) camera, as well as an external 0-8 W adjustable CW infrared laser (Hide-Wave Co., China). The NIR images are obtained by Si-based CCD using 750-900 nm band-pass filter. The SWIR images are obtained by InGaAs CCD using $1300 \mathrm{~nm}$ long-pass filter. The NIR/SWIR image and signal intensity are obtained by analysing NIR and SWIR images using professional software provided by Andor.

Stability and activity of Tm-rSiMo-AAO after storage. The Tm-rSiMo-AAO is stored under $-20{ }^{\circ} \mathrm{C}$ in DI water within 60 days. The protein-staining solution is freshly prepared by dissolving $20 \mathrm{mg}$ CBB in a mixture of 
$50 \mathrm{~mL}$ ethanol, $100 \mathrm{~mL} \mathrm{H}_{3} \mathrm{PO}_{4}$ and $850 \mathrm{~mL} \mathrm{DI}$ water. In a typical experiment, the freshly-prepared and stored Tm-rSiMo-AAO are separated via centrifugation $(8000 \mathrm{rpm})$ and washed with DI water three times. The supernatants and washing solutions are collected and $5 \mathrm{~mL}$ protein-staining solution is added, respectively. After 30 minutes, the absorbance of the above solutions at $595 \mathrm{~nm}$ (Abs.595) is determined to evaluate the stability of L-AAO on Tm-rSiMo. The freshly-prepared and stored Tm-rSiMo-AAO separated by centrifugation is then dispersed in $5 \mathrm{~mL}$ DI water and their absorbance at $800 \mathrm{~nm}(A b s .800)$ are determined respectively to evaluate the oxidation degree. To evaluate the activity of AAO on Tm-rSiMo after storage, $1 \mathrm{~mL}$ mimic L-AA working solution is added to both freshly-prepared and stored Tm-rSiMoAAO dispersion, respectively. After 40 minutes, the absorbance of Tm-rSiMo-AAO dispersions at $800 \mathrm{~nm}$ (Abs.800) is determined.

Influence of pH on activity of L-AAO. The $\mathrm{pH}$ of L-AAO solution is modulated by phosphate and diluted citric acid solution. The mimic L-AA working solution in freshly prepared according to a previous reported prescription (Supplementary table 1$)$. In a typical experiment, $10 \mu \mathrm{L} \mathrm{L-AAO}$ solution $\left(0.02 \mathrm{unit} \mathrm{mL}^{-1}\right)$ with various $\mathrm{pH}$ (4.0-7.0, at 0.5 intervals) is added to $90 \mu \mathrm{L}$ mimic L-AA working solution, HRP (3 units $\left.\mathrm{mL}^{-1}\right)$ and $O$-dianisidine $(10 \mu \mathrm{M})$. After 40 minutes, absorbance at $436 \mathrm{~nm}$ (Abs.436) of all samples is determined by monochromator-based multifunction microplate reader to evaluate the L-AAO activity, respectively.

Selective determination of L-AA by Tm-rSiMo-AAO. The control solution of ions (including $\mathrm{Na}^{+}, \mathrm{K}^{+}, \mathrm{Ca}^{2+}$, $\mathrm{Mg}^{2+}, \mathrm{Zn}^{2+}, \mathrm{Cl}^{-}, \mathrm{Br}^{-}, \mathrm{PO}_{4}{ }^{3-}$ and $\mathrm{CO}_{3}{ }^{2-}$ ) and biomolecules (including HSA, OOA, DOA, PTA, ODOA, ADA, 2-HA and $\mathrm{GlC}$ ) is freshly prepared and the concentration is 10 times higher than that of L-AA. The dissolution of fatty acids in DI water (including OOA, DOA, PTA, ODOA and ADA) is assisted by ethanol and ultrasonic. In a typical experiment, $1 \mathrm{~mL}$ mimic L-AA working solution and control solutions are added to $1 \mathrm{~mL} \mathrm{DI}$ water containing Tm-rSiMo-AAO $\left(2 \mathrm{mg} \mathrm{mL}^{-1}\right)$, respectively. After 40 minutes, the luminescence spectra of all samples are determined and analysed.

Ethics Statement. The fingerprints are collected from 10 males and 10 females in accordance to the official requirement of obtaining informed consent, in the form of a signature from each volunteer, acknowledging that they are aware of the procedure that will take place, any risks or benefits that may accompany the study, as well as acknowledging that they will not receive any payment for their participation. Informed consent from all volunteers who participates in this research study is obtained. After experiments, all fingerprints are thoroughly destroyed by dissolving to keep personal security.

Determination of L-AA extracted from fingerprints. The extraction of L-AA from fingerprints is performed by using a previous reported thermal/acid leaching protocol with reasonable modification. ${ }^{21}$ In a typical experiment, $120 \mu \mathrm{L} \mathrm{H}_{2} \mathrm{SO}_{4}$ solution $(0.01 \mathrm{M})$ is directly placed onto the fingerprint on a polyethylene chip. The chip is then heated to $40^{\circ} \mathrm{C}$ for 20 minutes. The leaching solution is collected off of the chip, diluted to $1 \mathrm{~mL}$ and used as the sample for determination after adjusting $\mathrm{pH}$ to 5.0. The determination process for fingerprint extraction is same to that for mimic L-AA working solution. 
Luminescence imaging of latent fingerprints. The Tm-rSiMo-AAO staining solution is freshly prepared by dispersing Tm-rSiMo-AAO in buffer solution of sodium citrate (SSC, $\mathrm{pH}=5.0$ ) containing Tween 20. The polyethylene chips for luminescence imaging are cleaned with DI water and dried in air before use. The fingerprints are used for staining procedure within 12 hours after collection. The fingerprints staining with Tm-rSiMo-AAO is referring to a typical protocol with modification. In a typical experiment, the samples are rinsed with DI water for 1 minute and then immersed in Tm-rSiMo-AAO staining solution under $40^{\circ} \mathrm{C}$ for 50 minutes with rotation. Then, NIR and SWIR imaging are performed and signals are analysed. ${ }^{8}$

Statistical Analysis. The NIR/SWIR ratiometric luminescence method is used to analyze 10 fingerprints collected from male and 10 fingerprints collected from female. Receiver operating characteristic (ROC) analysis was utilized to determine the forensic gender identification potential that the ability to correctly differentiate males and females by determination L-AA content in fingerprints. The ROC analysis captures the trade-off between sensitivity and specificity while changing a discrimination threshold, but it can be summarized as a single measurement (AUC). The sensitivity (true positive rate) was plotted against the specificity (true negative rate) in the ROC curve as a function of a variety of thresholds of class prediction probabilities. The overall accuracy depends on the overlap of the output signal distributions for the two classes, in this case, fingerprints of males and females. Values range between 0.5 and 1.0 , where a value of 0.5 indicates that the two distributions are identical and a value of 1.0 indicates that there is no overlap in the distributions of output signals for the two classes. The AUC is used as a lone measure of evaluating the efficiency of the model ranked subjects according to the probability assigned to the positive class. The AUC of the ROC curve was calculated by the trapezoidal method of integration with the corresponding $95 \%$ confidence intervals $(\mathrm{Cl})$.

\section{Declarations}

\section{Data availability}

The data that support the findings of this study are available from the authors on reasonable request.

\section{Acknowledgements}

The authors thank the funding of National Natural Science Foundation of China (82001945, 61905130), Natural Science Foundation of Beijing Municipality (KZ201810028045), Beijing Municipal Education Commission Outstanding Young Individual Project (CIT\&TCD201904082), Shanghai Pujiang Program (20PJ1410700), Youth High-level Talent Project of Capital Normal University (20530810024), Yanjing Young Scholar Program and Project of High-level Teachers in Beijing Municipal Universities in the Period of 13th Five-year Plan (IDHT20180517). Yuxin Liu personally thank the Max-Planck Society and MaxPlanck Institute for Colloids and Interfaces, as well as the support received from Prof. Peter Seeberger, Dr. Felix Loeffler, and Ms. Junfang Zhang. Yuxin Liu personally thank Dr. Xuejiao Wang and Dr. Hanlin Huang from Peking University for the pre-test and screening of UV-vis-NIR spectra of nanoprobes. Yuxin Liu and Zheng Wei also personally thank all people who provide their fingerprints and permission voluntarily. 


\section{Author contributions}

Y.L. and J.Z. conceived the project and wrote the original manuscript. X.Z. provided professional opinions on energy transfer and fingerprint imaging. J.Z. supervised the project. J.Z., X.Z., and L.L. obtained the funding for this research. Y.L. contributed to the synthesis of nanoprobe. Y.L. and Z.W. determined the electrochemical properties of nanoprobe. Y.L. and L. L. contributed to the determination of luminescence spectra. L.M. provided essential information and assistance in design and construction of nanoprobe. Y.L. designed and hold copyrights for all 3D images. All authors established the experiment schemes, performed the other experiments, analyzed the results, and wrote the manuscript.

\section{Competing financial interests}

The authors declare no competing financial interests.

\section{References}

1. Saferstein R. Criminalistics: An Introduction to Forensic Science. Pearson Prentice Hall Upper Saddle River, NJ (2007).

2. Houck MM, Siegel JA. Fundamentals of Forensic Science, 3rd Edition. Academic Press Ltd-Elsevier Science Ltd (2015).

3. Hazarika P, Russell DA. Advances in Fingerprint Analysis. Angew Chem Int Ed 51, 3524-3531 (2012).

4. Chavez D, Garcia CR, Oliva J, Diaz-Torres LA. A Review of Phosphorescent and Fluorescent Phosphors for Fingerprint Detection. Ceram Inter 47, 10-41 (2021).

5. Wang YL, et al. Real-Time Fluorescence in Situ Visualization of Latent Fingerprints Exceeding Level 3 Details Based on Aggregation-Induced Emission. J Am Chem Soc 142, 7497-7505 (2020).

6. Xi D, et al. A Facilely Synthesized Dual-State Emission Platform for Picric Acid Detection and Latent Fingerprint Visualization. Chem-Eur J 26, 2741-2748 (2020).

7. Hai J, et al. Reversible Response of Luminescent Terbium(lii)-Nanocellulose Hydrogels to Anions for Latent Fingerprint Detection and Encryption. Angew Chem Int Ed 57, 6786-6790 (2018).

8. Li JC, Zhu XJ, Xue M, Feng W, Ma RL, Li FY. Nd3+-Sensitized Upconversion Nanostructure as a DualChannel Emitting Optical Probe for near Infrared-to-near Infrared Fingerprint Imaging. Inorg Chem 55, 10278-10283 (2016).

9. Cadd S, Islam M, Manson P, Bleay S. Fingerprint Composition and Aging: A Literature Review. Sci Justi 55, 219-238 (2015).

10. Croxton RS, Baron MG, Butler D, Kent T, Sears VG. Variation in Amino Acid and Lipid Composition of Latent Fingerprints. Forens Sci Inter 199, 93-102 (2010).

11. Hadorn B, Hanimann F, Anders P, Curtius HC, Halverson R. Free Amino-Acids in Human Sweat from Different Parts of Body. Nature 215, 416-+ (1967). 
12. Brunelle $\mathrm{E}$, et al. Fingerprint Analysis: Moving toward Multiattribute Determination Via Individual Markers. Anal Chem 90, 980-987 (2018).

13. Brunelle E, et al. Coomassie Brilliant Blue G-250 Dye: An Application for Forensic Fingerprint Analysis. Anal Chem 89, 4314-4319 (2017).

14. Brunelle E, Huynh C, Le AM, Halámková L, Agudelo J, Halámek J. New Horizons for Ninhydrin: Colorimetric Determination of Gender from Fingerprints. Anal Chem 88, 2413-2420 (2016).

15. Liu YX, Wei Z, Liao X, Zhou J. Multichannel Lanthanide-Doped Nanoprobes Improve Diagnostic Performance. Acc Mater Res 1, 225-235 (2020).

16. Liu YX, Wei Z, Zhou J, Ma ZF. Simultaneous Multi-Signal Quantification for Highly Precise Serodiagnosis Utilizing a Rationally Constructed Platform. Nat Commun 10, 10 (2019).

17. Liu YX, et al. Customized Photothermal Therapy of Subcutaneous Orthotopic Cancer by MultiChannel Luminescent Nanocomposites. Adv Mater, DOI: 10.1002/adma.202008615 (2021).

18. Liu YX, et al. Optimization of Prussian Blue Coated NaDyF4:X\%Lu Nanocomposites for Multifunctional Imaging-Guided Photothermal Therapy. Adv Funct Mater 26, 5120-5130 (2016).

19. Zhang LY, et al. General Route to Multifunctional Uniform Yolk/Mesoporous Silica Shell Nanocapsules: A Platform for Simultaneous Cancer-Targeted Imaging and Magnetically Guided Drug Delivery. Chem-Eur J 18, 12512-12521 (2012).

20. Dou J, Zeng HC. Targeted Synthesis of Silicomolybdic Acid (Keggin Acid) inside Mesoporous Silica Hollow Spheres for Friedel-Crafts Alkylation. J Am Chem Soc 134, 16235-16246 (2012).

21. Huynh C, Brunelle E, Halámková L, Agudelo J, Halámek J. Forensic Identification of Gender from Fingerprints. Anal Chem 87, 11531-11536 (2015).

\section{Figures}
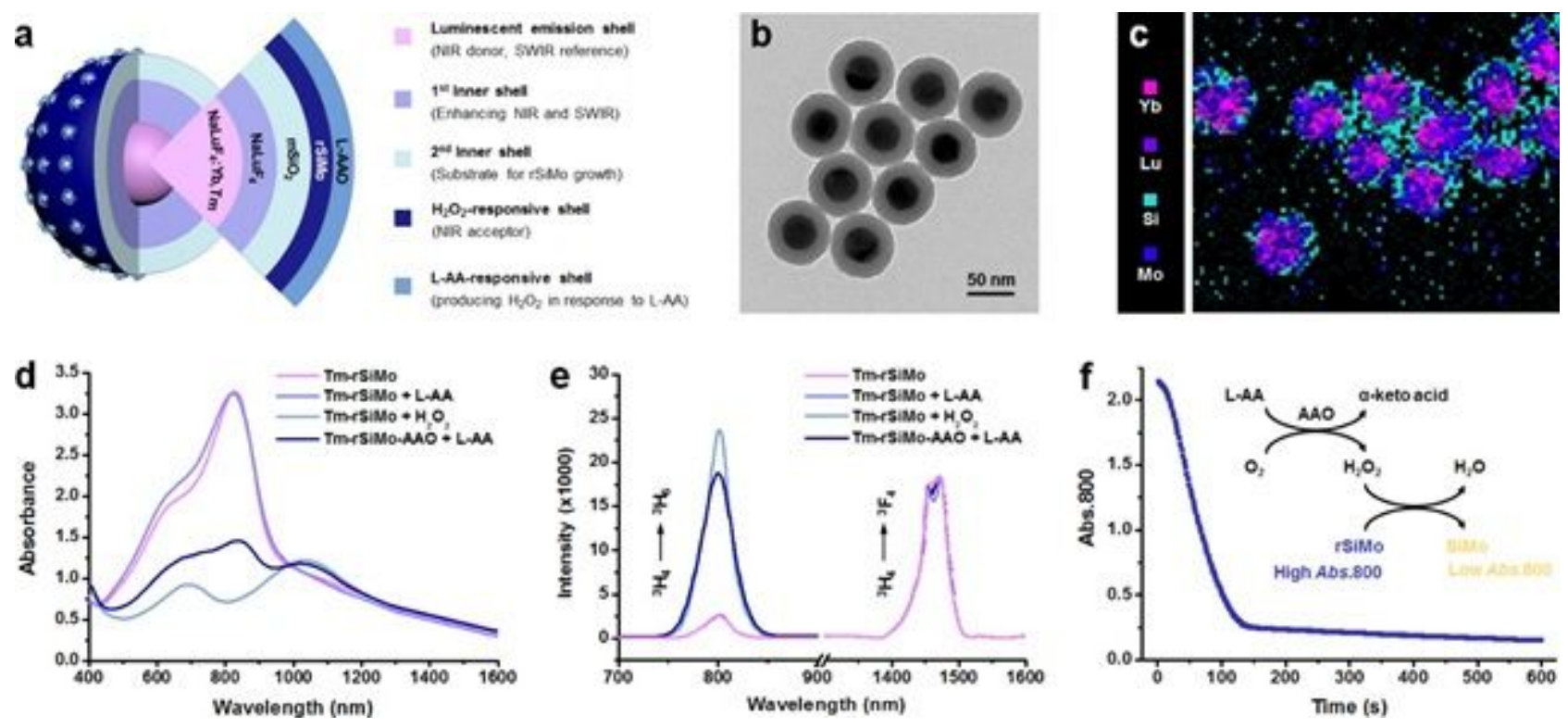

Figure 1 
Structure and characterization of Tm-rSiMo. a) Schematic diagram of the composition and function of each shell in an as-designed Tm-rSiMo. TEM image b) and EDX surface elements mapping result c) of Tm-rSiMo. UV-vis-NIR absorbance spectra d) and luminescence spectra e) of Tm-rSiMo and Tm-rSiMoAAO in L-AA solution. Tm-rSiMo in water and in $\mathrm{H} 2 \mathrm{O} 2$ solution are used as negative and positive control, respectively. f) Change of Abs. 800 of Tm-rSiMo-AAO as a function of time in presence of L-AA. Proposed mechanism for Abs.800 decreasing in responsive to L-AA is inserted.

a
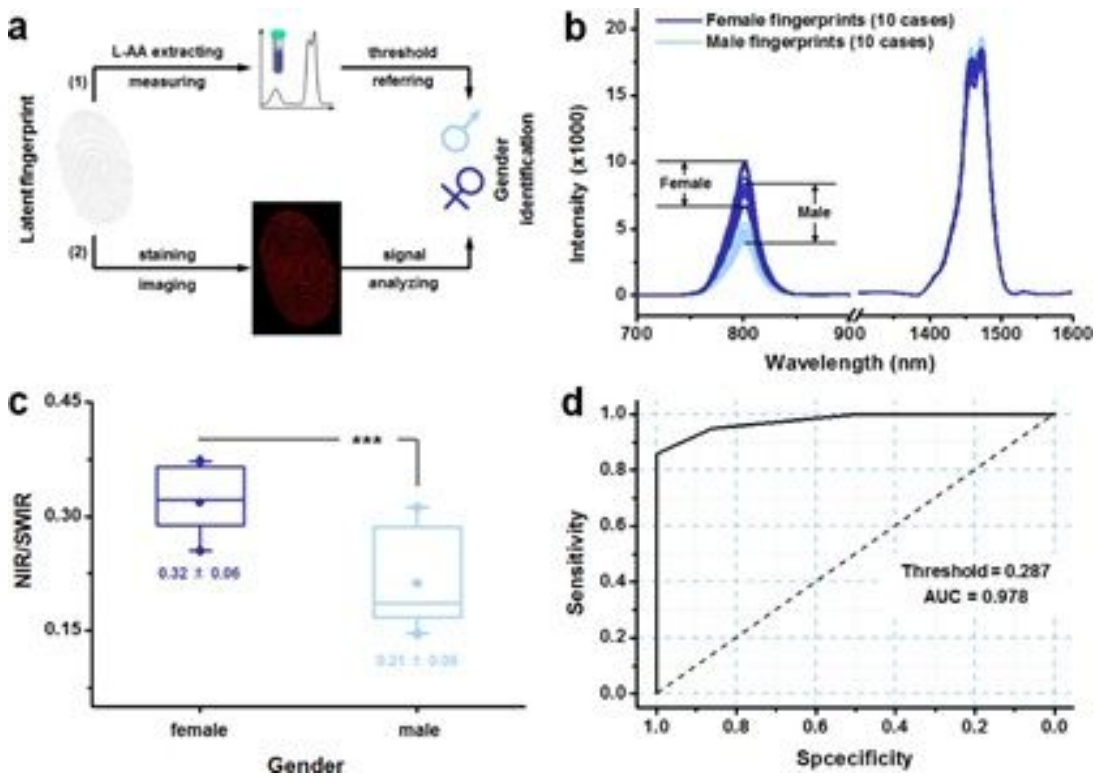

Figure 2

Gender identification by L-AA content. a) Schematic diagram of the forensic gender identification from fingerprints by two parallel procedures. Luminescence spectra $b$ ) and signal statistic $c$ ) of Tm-rSiMo-AAO in the solution of extract from fingerprints. The fingerprints are collected from 10 males and 10 females, respectively. d) Receiver operating characteristic (ROC) curve presenting the probability for the assay to correctly distinguish between male and female fingerprints based on the L-AA content in the fingerprints determined by luminescence spectra. Random choice is denoted by the black dotted diagonal line. Statistical significance was determined from one-way $t$ tests. ${ }^{\star \star \star} p<0.001$. 
a
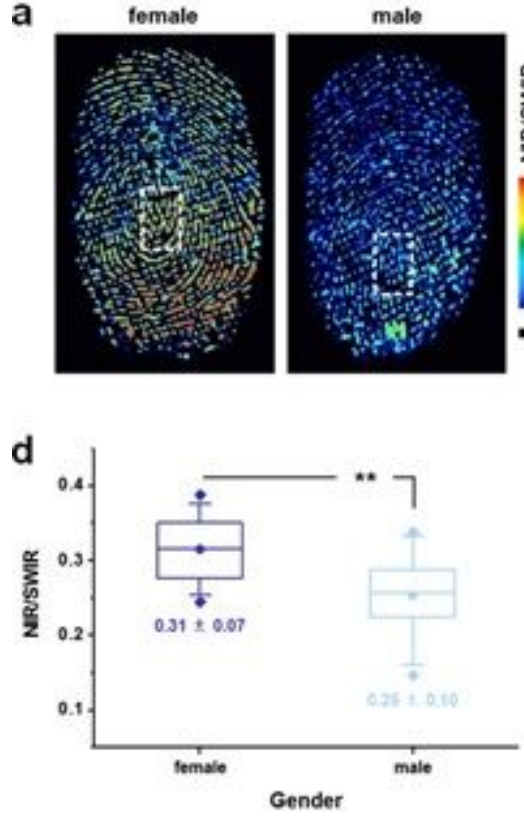

b

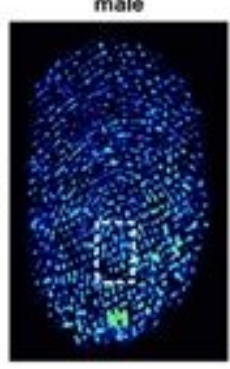

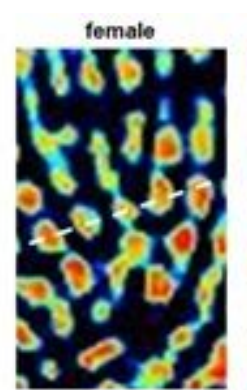

e

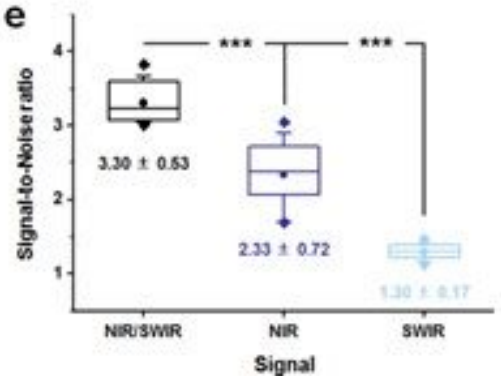

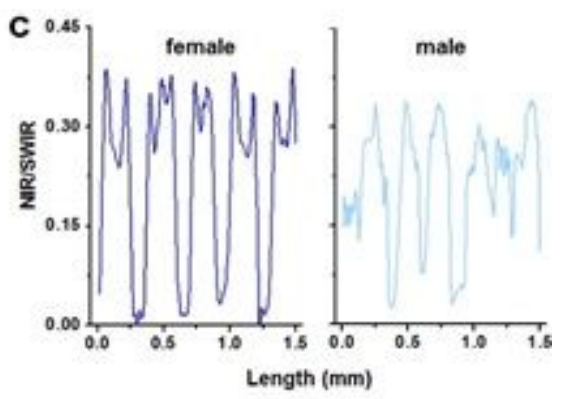

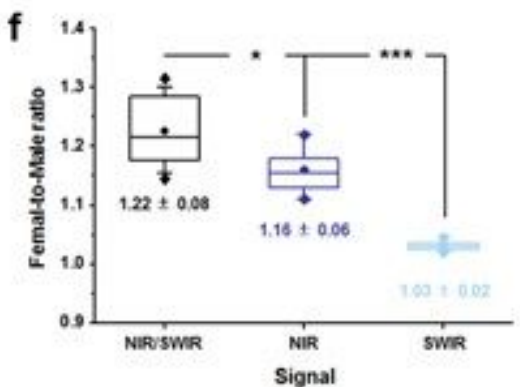

Figure 3

Gender identification by luminescence imaging. a) Colour-mapped NIR/SWIR ratiometric luminescence images a), local magnified images b), signal distribution $c$ ) and signal statistic d) of female and male fingerprint with Tm-rSiMo-AAO staining. The magnified region is denoted in a) by the white dotted square. The signal distribution pathway is denoted in b) by the white dotted diagonal line. Signal-to-noise ratio e) and female-to-male signal ratio f) determined from luminescence images by using NIR/SWIR, NIR, and SWIR signal. Statistical significance was determined from one-way $t$ tests. ${ }^{\star} p<0.05,{ }^{\star \star} p<0.01$, and ${ }^{* \star *} p$ $<0.001$.

\section{Supplementary Files}

This is a list of supplementary files associated with this preprint. Click to download.

- Supplementaryinformation20210408.docx 